\title{
Searching for light higgsinos with $b$-jets and missing leptons
}

\section{S. Bobrovskyi, F. Brümmer, W. Buchmüller and J. Hajer}

Deutsches Elektronen-Synchrotron DESY,

Notkestraße 85, 22607 Hamburg, Germany

E-mail: sergei.bobrovskyi@desy.de, felix.bruemmer@desy.de, wilfried.buchmueller@desy.de, jan.hajer@desy.de

ABSTRACT: A recently proposed class of supersymmetric models predicts rather light and nearly mass-degenerate higgsinos, while the other superparticles are significantly heavier. In this paper we study the early LHC phenomenology of a benchmark model of this kind. If the squarks and gluinos, and in particular the lighter stop, are still light enough to be within reach, then evidence for our model can be found in hadronic SUSY searches. Moreover, with dedicated searches it will be possible to distinguish the light higgsino model from generic SUSY models with a bino LSP. Search channels with $b$-jets and with isolated leptons play a crucial role for model discrimination.

Keywords: Supersymmetry Phenomenology

ArXIV EPRINT: 1111.6005 


\section{Contents}

$\begin{array}{llr}1 & \text { Introduction } & 1\end{array}$

2 Light higgsinos from higher-dimensional GUTs 2

3 Signatures 4

$\begin{array}{lll}4 & \text { Simulation of signal and background } & 7\end{array}$

5 Event selection and analysis $\quad 8$

$\begin{array}{lll}5.1 & \text { Discovery with all-hadronic search } & 8\end{array}$

5.2 Model discrimination: CMSSM-like models 9

$\begin{array}{ll}5.3 \text { Model discrimination: a simplified model } & 12\end{array}$

$\begin{array}{lll}6 & \text { Conclusions } & 15\end{array}$

\section{Introduction}

Recently a class of supersymmetric models was proposed [1] whose most characteristic feature is a large separation between the higgsino masses and the masses of the other superparticles. The particle content is that of the MSSM. Two higgsino-like neutralinos and a higgsino-like chargino are light: Their masses can be arbitrarily close to the direct chargino search bound from LEP, $m_{\chi_{1}^{ \pm}} \gtrsim 105 \mathrm{GeV}$. There is also a light Standard Modellike Higgs around $120 \mathrm{GeV}$. The heavier Higgs bosons, as well as the gaugino-like neutralinos and chargino, gluino, squarks and sleptons, have masses of at least $500 \mathrm{GeV}$, and possibly ranging up to several $\mathrm{TeV}$ (depending on the model details).

This kind of spectrum is naturally compatible with both LHC and LEP search bounds. Concerning the LHC, the absence of any signals for supersymmetry in cascade decays of first-generation squarks and gluinos points to them being rather heavy. On the other hand, evading the LEP bound on the lightest Higgs mass requires large loop corrections from third-generation soft terms, at least within the MSSM. This points to large thirdgeneration squark masses (bringing with them the inevitable fine-tuning which is present in the remaining parameter regions of the MSSM). Charginos and neutralinos, by contrast, can comfortably have masses between around only $100-200 \mathrm{GeV}$.

In the present paper we investigate the discovery potential of this scenario at the early LHC. If the only kinematically accessible states are indeed the light higgsinos (which is the case that was recently studied in some detail in [2]), the prospects tend to be poor. While higgsinos will be abundantly produced in electroweak processes at the LHC, any signals from their decays will be overwhelmed by Standard Model backgrounds. We therefore 
focus on the less extreme case where the mass separation between higgsinos and coloured superparticles is large, but not so large that the latter are altogether out of reach.

The lightest coloured superparticle in the models of [1] is always the right-handed scalar top quark. Stops decaying into higgsinos will consequently play the most important role in our study. ${ }^{1}$ Because of the large mass difference, this decay will give rise to very highenergetic jets, which provides a handle to distinguish signal events from Standard Model backgrounds. On the other hand, we can also discriminate between our light higgsino scenario and a generic MSSM with comparable squark and gluino masses. This is because in the latter one would expect to see also events with high-energetic isolated leptons from chargino and neutralino decays. Such events are absent in our scenario, since the higgsinolike chargino and neutralinos are nearly degenerate in mass; consequently, leptons in the final state are too soft to be detected.

This work is organized as follows. In section 2 we briefly review the class of models proposed in [1], and present the benchmark spectrum which our analysis will be based on. Section 3 contains a survey of the expected collider signatures. We comment on our analysis method in section 4, and present the results of our study in section 5. Finally, section 6 contains our conclusions.

\section{Light higgsinos from higher-dimensional GUTs}

The MSSM with light higgsinos and otherwise heavy superparticles has previously been studied e.g. in [8]. Recently some models were constructed which predict precisely this pattern, such as the "lopsided gauge mediation" models of $[9,10]$, as well as the mixed gauge-gravity mediation models of [1] which our analysis will be concerned with.

To briefly motivate the models of [1], we start by observing that, in certain grandunified models which naturally emerge from string constructions, there is a large number of vector-like states in incomplete GUT multiplets which should decouple close to the GUT scale. They serve as messengers for gauge-mediated supersymmetry breaking, inducing gaugino masses and scalar soft masses. Because of the high mediation scale, gravity mediation cannot be neglected. The gravity-mediated contributions to the MSSM parameters are however subdominant with respect to the gauge-mediated ones. The only exception are the $\mu$ and $B_{\mu}$ parameters, to which (minimal) gauge mediation does not contribute at all. These two parameters are induced by gravitationally suppressed interactions through the Giudice-Masiero mechanism, leading to light higgsinos and otherwise heavy superparticles. Related models with mixed gauge-gravity mediation have previously been discussed in [11].

The main properties of the higgsino sector can be summarized as follows. Since

$$
|\mu| \ll\left|M_{1}\right|,\left|M_{2}\right|,
$$

where $M_{1}$ and $M_{2}$ are the bino and wino masses respectively, there are three higgsino-like light states $\chi_{1}^{0}, \chi_{1}^{ \pm}$and $\chi_{2}^{0}$ with masses close to $|\mu|$. Their mass splittings will be of the order $|\mu|^{2} /\left|M_{1,2}\right|$, typically a few $\mathrm{GeV}$ for $|\mu| \gtrsim 100 \mathrm{GeV}$ and for TeV-scale gaugino masses.

\footnotetext{
${ }^{1}$ For some recent related studies of stops at the LHC, see for example [3-7].
} 
A $\chi_{1}^{0}$ LSP is not a viable dark matter candidate, since its relic density is extremely low due to efficient chargino coannihilation. This same mechanism, on the other hand, can substantially ameliorate the gravitino BBN problem if dark matter consists of gravitinos instead. The $\chi_{1}^{0}$ is then the NLSP, but it will be effectively stable on collider timescales.

The precise details of the spectrum depend on the messenger content of the model, on the exact choice of messenger scale and SUSY breaking scale, and on the assumptions about the gravity-mediated contributions to the soft terms. For our purposes of a first tentative study of collider phenomenology, it is convenient to adopt a simplified parametrization: We fix the gravitino mass to be $m_{3 / 2}=100 \mathrm{GeV}$, and choose a common messenger mass just below the GUT scale, $M_{\mathrm{m}}=5 \times 10^{15} \mathrm{GeV}$. Then the essential free parameters are the gaugino masses $M_{1}, M_{2}$ and $M_{3}$, the Higgs soft mass mixing $B_{\mu}$, and the higgsino mass $\mu$. At the GUT scale we expect $\left|B_{\mu}\right| \simeq|\mu|^{2} \simeq m_{3 / 2}^{2}$ and $\left|M_{1,2,3}\right| \gg m_{3 / 2}$. Scalar soft masses are dominated by the gauge-mediated contribution, which is completely fixed after prescribing the gaugino masses. Explicitly, they are given by the standard minimal gauge mediation formula

$$
m_{\Phi}^{2}=2\left(\frac{g^{2}}{16 \pi^{2}}\right)^{2}\left(\sum_{a} C_{a} n_{a}\right)\left|\frac{F}{M_{\mathrm{m}}}\right|^{2},
$$

where $a=1,2,3$ labels the Standard Model gauge factors, $C_{a}$ is the corresponding quadratic Casimir of $\Phi$, the SUSY breaking scale $F$ is

$$
F=\sqrt{3} m_{3 / 2} M_{\text {Planck }}=\left(2 \times 10^{10} \mathrm{GeV}\right)^{2},
$$

and the effective messenger numbers $n_{a}$ are obtained by inverting the standard gaugino mass formula

$$
M_{a}=\frac{g^{2}}{16 \pi^{2}} n_{a} \frac{F}{M_{\mathrm{m}}} .
$$

We are neglecting the running of the gauge couplings between $M_{\mathrm{m}}$ and $M_{\mathrm{GUT}}$, as well as the subdominant gravity-mediated contributions. Trilinear terms are again dominated by gravity mediation; for simplicity we choose them to be universal and set $A_{0}=\mu$.

Having thus fixed the MSSM parameters at the messenger scale, we evolve them to the weak scale by means of their renormalization group equations using SOFTSUSY [12]. Reproducing the correct value of the $Z$ mass further reduces the number of free parameters by one. In the end, within our simplified ansatz the mass spectrum is entirely determined by the five parameters $M_{1}, M_{2}, M_{3}, \mu$ and $B_{\mu}$ at the messenger scale. These are subject to the conditions that electroweak symmetry should be broken with $m_{Z}=91 \mathrm{GeV}$, and that there should be a separation of mass scales according to

$$
\mu \sim \sqrt{B_{\mu}} \sim m_{3 / 2} \ll M_{1} \sim M_{2} \sim M_{3}
$$

Table 1 shows two examples for low-energy spectra, both with $\mu=150 \mathrm{GeV}$ and $\sqrt{B_{\mu}}=200 \mathrm{GeV}$ and with equal values for $M_{1}$ and $M_{2}$. Spectrum I has $M_{1}=M_{2}=$ $1250 \mathrm{GeV}$ and $M_{3}=428 \mathrm{GeV}$; these parameters are chosen such that the model is close to the present LHC exclusion limits. Spectrum II has $M_{1}=M_{2}=3 \mathrm{TeV}$ and $M_{3}=1130 \mathrm{GeV}$, for which the model would be invisible at the early LHC and quite difficult to find even 


\begin{tabular}{|crrrrr|}
\hline particle & \multicolumn{5}{c|}{ model } \\
& Spectrum I & Spectrum II & HH50 & HH50 & simplified \\
\hline$h_{0}$ & 116 & 121 & 115 & 117 & 117 \\
\hline$\chi_{1}^{0}$ & 124 & 117 & 206 & 207 & 125 \\
$\chi_{1}^{ \pm}$ & 129 & 119 & 389 & 395 & \\
$\chi_{2}^{0}$ & 134 & 121 & 389 & 395 & \\
\hline$\chi_{3}^{0}$ & 559 & 1319 & 635 & 771 & \\
$\chi_{4}^{0}$ & 1059 & 2453 & 649 & 778 & \\
$\chi_{2}^{ \pm}$ & 1059 & 2453 & 648 & 779 & \\
\hline$H_{0}$ & 641 & 660 & 861 & 958 & \\
$A_{0}$ & 642 & 666 & 861 & 958 & \\
$H^{ \pm}$ & 648 & 672 & 865 & 962 & \\
\hline$\tilde{g}^{ \pm}$ & 1063 & 2485 & 1167 & 1167 & \\
\hline$\tilde{t}_{1}$ & 665 & 1558 & 860 & 660 & 659 \\
$\tilde{b}_{1}$ & 797 & 1614 & 1034 & 943 & \\
$\tilde{u}_{1}$ & 1155 & 2438 & 1122 & 1130 & \\
$\tilde{d}_{1}$ & 1065 & 2294 & 1119 & 1127 & \\
\hline$\tilde{\tau}_{1}$ & $1070-1500$ & $2300-3100$ & $1120-1160$ & $990-1270$ & \\
\hline other squarks & 509 & 669 & 528 & 520 & \\
\hline other sleptons & $790-1160$ & $1400-2300$ & $530-600$ & $530-600$ & \\
\hline
\end{tabular}

Table 1. A light and a heavy spectrum, with a CMSSM point HH50, a CMSSM-like point HH50' and a simplified model for comparison. The parameters defining these models are listed in table 2 . Particle masses are in $\mathrm{GeV}$.

at $14 \mathrm{TeV}$. Our analysis will be mostly concerned with the phenomenology of Spectrum I at $\sqrt{s}=7 \mathrm{TeV}$.

For comparison, we have also included a similar CMSSM benchmark point HH50 and a CMSSM-like benchmark point HH50'. HH50 has $m_{0}=M_{1 / 2}=500 \mathrm{GeV}, \tan \beta=10$, $\operatorname{sign}(\mu)=+1$ and $A_{0}=0$. HH50' is defined in the same way, but with the soft terms of the third generation chosen differently: Third-generation squarks and sleptons were given a universal soft mass $m_{0}^{(3)}=300 \mathrm{GeV}$ and a trilinear $A$-parameter $A_{0}^{(3)}=-1 \mathrm{TeV}$. This choice was made in order to have a reference spectrum whose $\tilde{t}_{1} \tilde{t}_{1}^{*}$ production cross section is comparable to that of Spectrum I, while closely resembling the CMSSM. Finally, we also list a comparable simplified model, containing only the $\tilde{t}_{1}$ and a bino-like neutralino LSP. The model definitions are summarized in table 2.

\section{Signatures}

The light higgsinos of our scenario will be produced in copious numbers in electroweak processes at the LHC. The Drell-Yan process gives rise to $\chi_{1}^{+} \chi_{1}^{-}, \chi_{1}^{ \pm} \chi_{1,2}^{0}$ and $\chi_{1}^{0} \chi_{2}^{0}$ final states, and $W$ boson fusion can give like-sign $\chi_{1}^{ \pm} \chi_{1}^{ \pm}$pairs. The subsequent decays of $\chi_{2}^{0}$ and $\chi_{1}^{ \pm}$into $\chi_{1}^{0}$ will lead to events with missing energy and soft jets or leptons. 


\begin{tabular}{|lrrrrrrrrr|}
\hline model & $\mu$ & $\sqrt{B_{\mu}}$ & $M_{1}=M_{2}$ & $M_{3}$ & $m_{0}$ & $m_{0}^{(3)}$ & $A_{0}$ & $A_{0}^{(3)}$ & $\tan \beta$ \\
\hline Spectrum I & 150 & 200 & 1250 & 428 & & & & & 46 \\
Spectrum II & 150 & 200 & 3000 & 1130 & & & & & 53 \\
\hline HH50 & & & 500 & 500 & 500 & 500 & 0 & 0 & 10 \\
HH50' & & & 500 & 500 & 500 & 300 & 0 & -1000 & 10 \\
\hline
\end{tabular}

Table 2. Defining parameters for a light and a heavy spectrum, with a CMSSM point and a CMSSM-like point for comparison. Particle masses are in $\mathrm{GeV}$. In HH50' third-generation squarks and sleptons were given a universal soft mass $m_{0}^{(3)}$ and a trilinear $A$-parameter $A_{0}^{(3)}$.
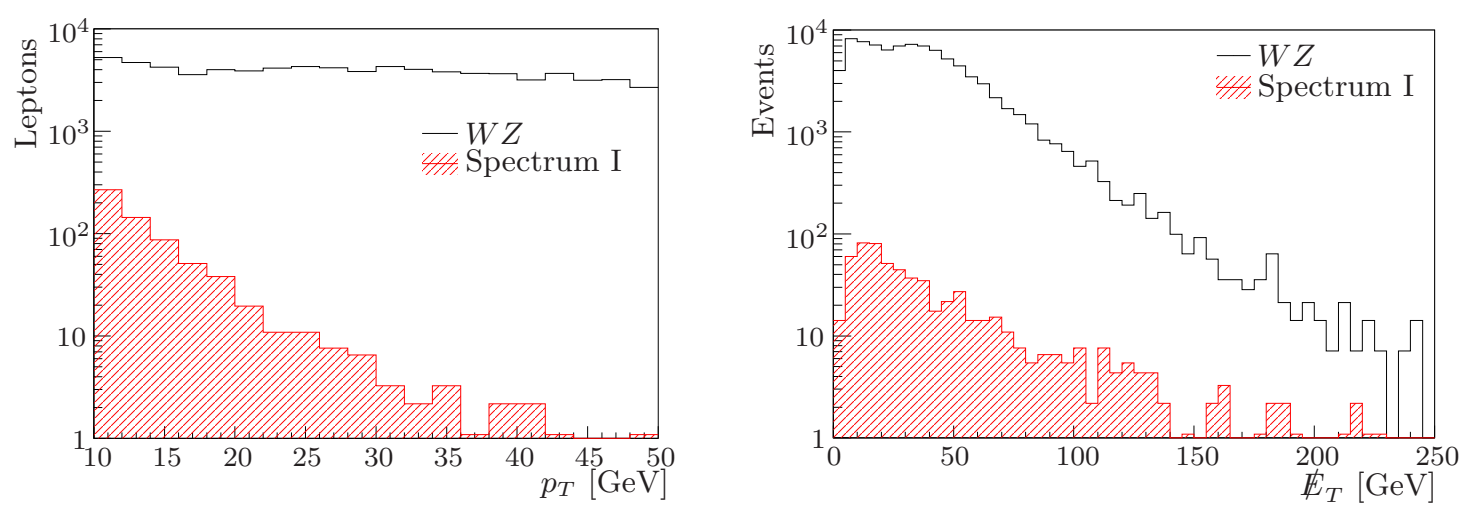

Figure 1. Lepton transverse momentum and missing transverse energy distributions of leptonic events from higgsino decays with Spectrum I. The higgsinos are produced in electroweak processes; the numbers are scaled to $20 \mathrm{fb}^{-1}$. For comparison, the SM background from $W Z$ production (which is just one of the several contributing processes) is also shown. See section 4 for details of the event simulation.

Unfortunately, with the higgsino mass splittings in the range of only a few $\mathrm{GeV}$, most of these jets and leptons are too soft to even trigger on, and those events with high enough $p_{T}$ to be detected are completely swamped by the Standard Model background. Demanding large missing transverse energy does not help much, since also the $\mathbb{E}_{T}$ spectrum falls very rapidly. For illustration, the lepton $p_{T}$ and $\mathbb{E}_{T}$ distributions for Spectrum I are shown in figure 1. We have also studied events with additional jets from initial-state gluon radiation, in order to increase the number of events with larger $p_{T}$ and $\mathbb{E}_{T}$. While this somewhat enhances the tails of the distributions, it also reduces the overall cross section, and the combined effect does very little to improve the overall situation. In conclusion we confirm the findings of [2] that, in order to find evidence for our scenario in electroweak processes, a linear collider would be far better suited. ${ }^{2}$

We are therefore led to consider those regions of parameter space where some coloured

\footnotetext{
${ }^{2}$ For the LHC, a monojet (from initial-state gluon radiation) together with large missing $E_{T}$ might perhaps be a useful signal, in combination with other searches. We will however not pursue this possibility in the present work because of the difficulties in accurately estimating the background without a full detector simulation.
} 
superparticles are still light enough to be produced at the LHC. The lightest coloured superparticle in our class of models is always the lighter of the scalar top quarks $\tilde{t}_{1}$. At the LHC it may be produced in pairs, or it may appear in cascade decays of first-generation squarks and gluinos if these are kinematically accessible. It turns out that processes involving the $\tilde{t}_{1}$ are particularly well suited to find evidence for our scenario (or to constrain it), and also to distinguish it from more generic incarnations of the MSSM.

For definiteness we will from now on focus on the Spectrum I benchmark point $M_{1}=$ $M_{2}=1250 \mathrm{GeV}, M_{3}=428 \mathrm{GeV}, \mu=150 \mathrm{GeV}, \sqrt{B_{\mu}}=200 \mathrm{GeV}$. In a sense this is a maximally optimistic set of parameters, chosen such that it is still marginally allowed by current search limits. We are planning to extend our analysis to cover a wider range of parameter space in the future.

With superparticle masses as in Spectrum I, the clearest signatures at the early LHC will be jets with missing $E_{T}$. We will see that the cross sections for stop pair production on the one hand and the more familiar $\tilde{q} \tilde{q}, \tilde{q} \tilde{q}^{*}, \tilde{q} \tilde{g}$ and $\tilde{g} \tilde{g}$ production (where $\tilde{q}$ stands for any first-generation squark) on the other hand are comparable; all these processes contribute to the signal.

More importantly, once there is evidence for supersymmetry in searches for jets plus missing $E_{T}$, our model can also be distinguished experimentally from generic variants of the MSSM which lack its characteristic features of light and near-degenerate higgsinos. This is achieved by focussing on the stop pair production channel. In Spectrum I, stop decays do not involve hard leptons, since possible leptons from $\chi_{2}^{0}$ or $\chi_{1}^{ \pm}$decays are too soft to be detected. The signature of a $\tilde{t}_{1}$ is therefore always a hard $b$-jet plus missing $E_{T}$; a typical stop pair event is shown in figure 2. By contrast, in generic supersymmetric models one usually expects also events with jets, missing $E_{T}$ and isolated leptons, be it from cascade decays of squarks and gluinos or from $\tilde{t}$ decaying into charginos or non-LSP neutralinos. Once a signal is found in the jets + MET channel, we could use the absence of signals with leptons to severely constrain interpretations in terms of generic supersymmetry, thus providing further indirect evidence for our scenario.

We may even be able to discriminate between our model and a "simplified model" comprising only a $\tilde{t}_{1}$ and a bino-like $\chi_{1}^{0}$. In such a framework, likewise, no events with hard isolated leptons are expected. However, because the only possible $\tilde{t}_{1}$ decay is then $\tilde{t}_{1} \rightarrow t \chi_{1}^{0}$ with the $t$ decaying further into $b W$, the $b$-jet spectrum turns out to be significantly different from that of our model, where about half of the stops decay directly into a $b$ quark without an intermediate top.

In the following sections we present the results of three simulated searches. The first is for jets and large missing $E_{T}$, in order to show that early LHC will be able to find evidence for our model. The second also includes leptons, to show that early LHC will, furthermore, be able to distinguish our model from a comparable CMSSM-like model. More precisely, our model will be compared both with the CMSSM point HH50, which has similar $\tilde{g}$ and $\tilde{q}$ production cross sections, and with the CMSSM-like point HH50', which in addition has also a comparable $\tilde{t}_{1}$ pair production cross section. Finally, we present a search with the cuts optimized to select events from $\tilde{t}_{1}$ pair production, and compare the result with the simplified model mentioned above. 


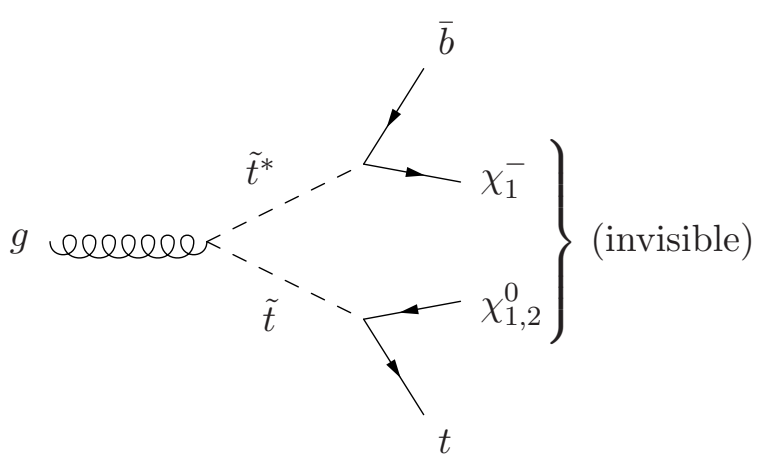

Figure 2. An example for a stop pair production event, showing up as to two high-energetic $b$-jets and missing energy.

\begin{tabular}{|lccccccc|}
\hline model & $\sigma\left(\tilde{q} \tilde{q}^{*}\right)$ & $\sigma(\tilde{q} \tilde{q})$ & $\sigma\left(\tilde{t} \tilde{t}^{*}\right)$ & $\sigma\left(\tilde{b} \tilde{b}^{*}\right)$ & $\sigma(\tilde{g} \tilde{g})$ & $\sigma(\tilde{g} \tilde{q})$ & $\sigma($ tot $)$ \\
\hline Spectrum I & 0.388 & 3.83 & 5.61 & 0.6 & 2.9 & 8.45 & 21.78 \\
HH 50 & 1.79 & 12 & 0.682 & 0.044 & 1 & 9.3 & 24.8 \\
HH 50' & 1.65 & 11.5 & 5.96 & 0.136 & 0.979 & 8.9 & 29 \\
\hline
\end{tabular}

Table 3. Production cross sections of different models in fb calculated with PROSPINO. The cross section for $\tilde{b} \tilde{b}^{*}$-production is given at the lowest order, all other cross sections are calculated at NLO.

\section{Simulation of signal and background}

All Monte Carlo samples were generated with MADGRAPH 4.4.44 [13] interfaced with PYTHIA 6.4.22 [14] using CTEQ6L1 parton distribution functions [15]. In order to generate signal events, decay widths of supersymmetric particles were computed with SDECAY [16] from spectra calculated with SOFTSUSY. The generic detector simulation DELPHES [17], tuned to the CMS detector, was used in order to account for effects of event reconstruction at the detector level.

The signal production cross sections are listed in table 3. For Spectrum I 43500 signal events were simulated, to be compared with 435 events expected at the early LHC with an assumed integrated luminosity yield of $20 \mathrm{fb}^{-1}$. The number of signal events passing the cuts should therefore eventually be divided by a normalization factor 100 for a realistic estimate. For HH50 and HH50', we simulated 10000 events each, with respectively 496 and 580 events expected, so the normalization factors are 20 and 17 respectively.

The corresponding figures for Standard Model backgrounds are listed in table 4. It turns out that $t \bar{t}$ is the most important background. Since, consequently, the best statistics is needed for this channel, we have simulated about three times more events than expected. For the remaining backgrounds, the number of simulated events roughly matches the number of expected events, or exceeds it in the case of tri-bosons (where the cross sections are small) in order to avoid large Monte Carlo errors. An exception are background events with vector bosons plus jets, where we have only simulated a small fraction of the expected events. However, as will become clear when we present the cut flows, this background is very efficiently removed by our cuts. Therefore it can be safely neglected without having to simulate the full sample. 


\begin{tabular}{|lrrr|}
\hline \multirow{2}{*}{ sample } & $\sigma$ in $\mathrm{pb}$ & \multicolumn{2}{c|}{ events } \\
\cline { 3 - 4 } & & \multicolumn{1}{c|}{ expected } & simulated \\
\hline$t \bar{t}$ & 163 & $3.3 \times 10^{6}$ & $11.3 \times 10^{6}$ \\
single top & 85.1 & $1.7 \times 10^{6}$ & $1.7 \times 10^{6}$ \\
$W+$ jet & 826 & $1652 \times 10^{4}$ & $5 \times 10^{4}$ \\
$W^{+} W^{-}$ & 44.974 & $899 \times 10^{3}$ & $1000 \times 10^{3}$ \\
$W^{+} Z$ & 11.580 & $358 \times 10^{3}$ & $400 \times 10^{3}$ \\
$W^{-} Z$ & 6.342 & 350 & \\
$Z Z$ & 6.195 & $124 \times 10^{3}$ & $150 \times 10^{3}$ \\
$W^{+} W^{-} W^{+}$ & $4 \times 10^{-2}$ & 800 & 15000 \\
$W^{+} W^{-} Z$ & $3 \times 10^{-2}$ & 600 & 15000 \\
$W^{+} Z Z$ & $9 \times 10^{-3}$ & 180 & 15000 \\
$Z Z Z$ & $3 \times 10^{-3}$ & 60 & 5629 \\
\hline
\end{tabular}

Table 4. Cross sections and numbers of generated events of SM background used in the present analysis. The single top production cross-section includes all LHC production channels. The cross sections for the tri-boson events are calculated at the Born level with MADGRAPH, all other cross sections are taken from $[18-20]$

\section{$5 \quad$ Event selection and analysis}

\subsection{Discovery with all-hadronic search}

The first analysis serves to show that LHC will be able to find evidence for our model, i.e. to distinguish its signatures from the Standard Model background.

In the first stage, candidate events with multiple high-energetic jets and missing transverse energy are selected with the following pre-selection cuts at the level of the detector simulation:

- $1<N(j)<5$, where $p_{T}(j)>100 \mathrm{GeV}$,

- $\mathbb{E}_{T}>50 \mathrm{GeV}$.

Furthermore, all events with an isolated lepton (electron or muon) with $p_{T}>10 \mathrm{GeV}$ are rejected in order to suppress events with genuine missing energy from neutrinos:

- $N(l)=0$.

After imposing these pre-selection cuts, we use a set of cuts optimized for discriminating between signal and background. Events are required to satisfy

- $H T^{\prime}>500 \mathrm{GeV}$,

where $H T^{\prime}$ is the sum of the transverse momenta of the two most energetic jets,

$$
H T^{\prime}=\sum_{i=1}^{2} p_{T}\left(j_{i}\right) .
$$


Following the experimental analyses, we use the $\alpha_{T}$ variable [21-23] as the main discriminator against QCD multi-jet production, defined for di-jet events as:

$$
\alpha_{T}=\frac{E_{T}\left(j_{2}\right)}{M_{T}}=\frac{E_{T}\left(j_{2}\right)}{\sqrt{\left(\sum_{i=1}^{2} E_{T}\left(j_{i}\right)\right)^{2}-\left(\sum_{i=1}^{2} p_{x}\left(j_{i}\right)\right)^{2}-\left(\sum_{i=1}^{2} p_{y}\left(j_{i}\right)\right)^{2}}},
$$

where $j_{2}$ denotes the next-to-leading jet. In our analysis we use $p_{T}$ of the jets provided by DELPHES instead of $E_{T}$, and require the event to have

- $\alpha_{T}>0.55$

in order to pass the cut. In events with jet multiplicity $N(j)>2$, two pseudo jets are formed following the CMS strategy [23] and the $\alpha_{T}$ variable is constructed from the pseudojets. Finally, in order to further suppress the $t \bar{t}$ background, we demand a very high value of missing transverse energy:

- $\mathbb{E}_{T}>400 \mathrm{GeV}$.

Because of the high $\mathbb{E}_{T}$ cut in combination with the selection based on $\alpha_{T}$, we can safely neglect QCD di- and multi-jet background contributions. The resulting cut flow is shown in table 5 .

Evidently, with this analysis it will be possible to discriminate between our model and the Standard Model background. The same is true for the HH50 and HH50' models. This result is of course unsurprising, since all these benchmark points were chosen to lie near the $1 \mathrm{fb}^{-1}$ exclusion bounds, and here we are assuming a data sample of $20 \mathrm{fb}^{-1}$.

\subsection{Model discrimination: CMSSM-like models}

The more interesting question is that of model discrimination. For this a fully hadronic search such as the one we just presented is not suitable, even though the number of events passing the above cuts is significantly different between our model and HH50 / HH50'. This difference could, after all, be accounted for by slightly different squark and gluino production cross sections - for instance, the HH50 and HH50' spectra would just need to be slightly heavier in order to reproduce the 42 events after cuts which we found for our model.

In fact, some information can be gained already by requesting, in addition to the cuts of section 5.1, that at least one jet should be $b$-tagged. We assume a $p_{T}$-independent $b$ tagging efficiency of $40 \%$, and a mistagging probability of $10 \%$ as implemented in DELPHES. The additional cut is then

- $N(b$-jets $) \geq 1$.

The cut flow is shown in table 6. Note that the number of events from both HH50 and HH50' is dramatically reduced. This is partly because, in our model, a sizeable fraction of events was due to $\tilde{t}$ pair production, and the gluino can only decay into $\tilde{t}_{1}$ or $\tilde{b}_{1}$. By contrast, in HH50 and HH50' most events involve $\tilde{q}$ decays which do not necessarily lead to 


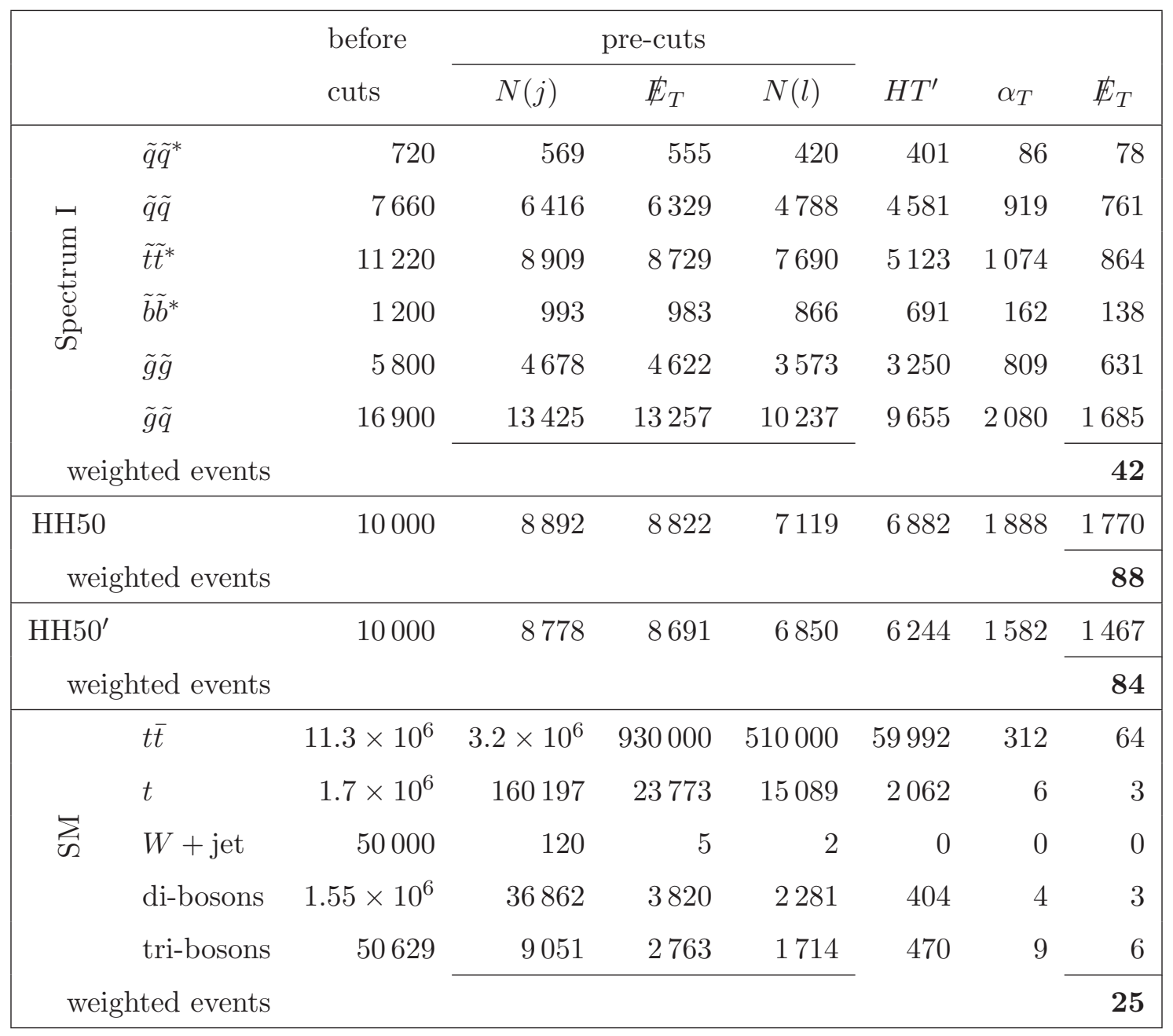

Table 5. Cut flow of general all-hadronic analysis for different signals and backgrounds at $\sqrt{s}=$ $7 \mathrm{TeV}$. Figures are given for all events that were simulated. The bold numbers are the events surviving all cuts, properly normalized to an integrated luminosity of $20 \mathrm{fb}^{-1}$. The cut flow for the Spectrum I is shown separately for each different production channel.

$b$-jets. Moreover, by vetoing events with isolated leptons, fewer $\tilde{t}_{1}$ events in our model are cut away than in HH50 and HH50' - these models tend to produce more leptonic events, which we will now put to use in a separate semi-leptonic analysis.

More precisely, as explained in section $3, \tilde{t}_{1}$ decays in our model can give hard isolated leptons at most from secondary top decays (which is, incidentally, also true for $\tilde{b}_{1}$ and even $\tilde{g}$ decays, since the gluino can only decay into $\tilde{t}_{1}$ or $\tilde{b}_{1}$ ). In HH50 and HH50' many more leptons are expected, jets with missing $E_{T}$ and isolated leptons being one of the hallmark signatures for generic supersymmetry. This motivates a semi-leptonic search for better model discrimination.

An event is selected for further analysis if it contains exactly one lepton (muon or electron) candidate

- $N(l)=1, \quad p_{T}(l)>15 \mathrm{GeV}$. 


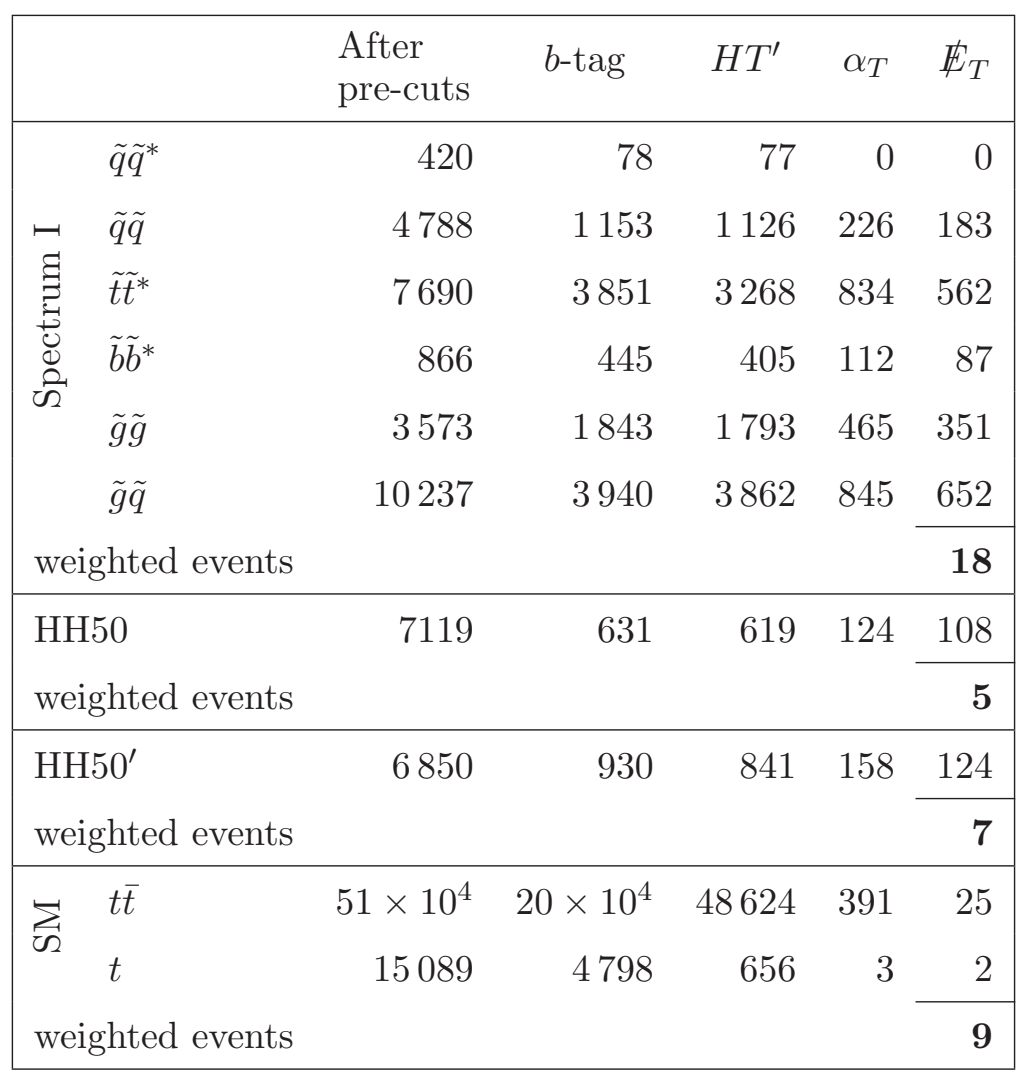

Table 6. Cut flow of the hadronic analysis with $b$-tagging for different signals and the relevant backgrounds at $\sqrt{s}=7 \mathrm{TeV}$. The remaining signal and background events, scaled to an integrated luminosity of $20 \mathrm{fb}^{-1}$, are printed in bold. The cut flow for Spectrum I is shown separately for each different production channel.

Other than that, our pre-selection cuts are as before,

- $N(j)>1, \quad p_{T}(j)>100 \mathrm{GeV}$,

- $\mathbb{E}_{T}>50 \mathrm{GeV}$.

The actual cuts are now as follows. We select events with exactly two high-energetic jets,

- $N(j)=2$.

This criterion selects preferably the $\tilde{t} \tilde{t}^{*}$ production channel, since usually more than two jets are expected to appear in channels involving $\tilde{q}$ or $\tilde{g}$. Furthermore, we employ the transverse mass variable

$$
m_{T}=\sqrt{2 p_{T}(l) \mathbb{E}_{T}\left(1-\cos \Delta \phi\left(l, \mathbb{E}_{T}\right)\right)},
$$

where $\Delta \phi\left(l, \mathbb{E}_{T}\right)$ is the angle between missing transverse energy and the momentum of the lepton in the transverse plane. This variable is bounded by the $W$-boson mass if the lepton and $\mathbb{E}_{T}$ originate in $W$-boson decay. We select events with

- $m_{T}>100 \mathrm{GeV}$, 


\begin{tabular}{|c|c|c|c|c|c|c|c|c|c|c|}
\hline & \multirow{2}{*}{$\begin{array}{l}\text { before } \\
\text { cuts }\end{array}$} & \multicolumn{3}{|c|}{ pre-cuts } & \multirow[b]{2}{*}{$N(j)$} & \multirow[b]{2}{*}{$m_{T}$} & \multirow[b]{2}{*}{ Iso } & \multirow[b]{2}{*}{$H T^{\prime}$} & \multirow[b]{2}{*}{$\mathbb{E}_{T}$} \\
\hline & & & $N(l)$ & $N(j)$ & $\mathbb{E}_{T}$ & & & & & \\
\hline \multirow{7}{*}{ 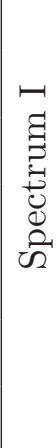 } & $\tilde{q} \tilde{q}^{*}$ & 720 & 238 & 233 & 229 & 26 & 17 & 6 & 6 & 1 \\
\hline & $\tilde{q} \tilde{q}$ & 7660 & 2690 & 2650 & 2622 & 380 & 271 & 129 & 123 & 74 \\
\hline & $\tilde{t} \tilde{t}^{*}$ & 11220 & 4063 & 3202 & 3135 & 2191 & 1701 & 230 & 90 & 40 \\
\hline & $\tilde{b} \tilde{b}^{*}$ & 1200 & 449 & 367 & 367 & 244 & 180 & 25 & 16 & 8 \\
\hline & $\tilde{g} \tilde{g}$ & 5800 & 2224 & 2202 & 2173 & 258 & 207 & 53 & 46 & 29 \\
\hline & $\tilde{g} \tilde{q}$ & 16900 & 6397 & 6346 & 6261 & 690 & 536 & 170 & 142 & 76 \\
\hline & vents & & & & & & & & & 2 \\
\hline \multirow{2}{*}{\multicolumn{2}{|c|}{$\begin{array}{l}\text { HH50 } \\
\text { events }\end{array}$}} & 10000 & 2432 & 2352 & 2330 & 615 & 438 & 242 & 225 & 147 \\
\hline & & & & & & & & & & 7 \\
\hline \multirow{2}{*}{\multicolumn{2}{|c|}{$\begin{array}{l}\text { HH50' } \\
\text { events }\end{array}$}} & 10000 & 2699 & 2519 & 2496 & 796 & 576 & 308 & 246 & 147 \\
\hline & & & & & & & & & & 9 \\
\hline \multirow{2}{*}{\multicolumn{2}{|c|}{$\begin{array}{l}\mathrm{SM} t \bar{t} \\
\text { events }\end{array}$}} & $11 \times 10^{6}$ & $4 \times 10^{6}$ & $1 \times 10^{6}$ & 440000 & 350000 & 45584 & 29942 & 1266 & 3 \\
\hline & & & & & & & & & & 1 \\
\hline
\end{tabular}

Table 7. Cut flow of semi-leptonic analysis for different signals and relevant background at $\sqrt{s}=7 \mathrm{TeV}$. The remaining signal and background events, scaled to an integrated luminosity of $20 \mathrm{fb}^{-1}$, are printed in bold. The cut flow for Spectrum I is shown separately for each different production channel.

and ensure that the leptons in these events are isolated. Furthermore, as in the previous analysis we demand that the two jets have high transverse momentum and high missing transverse energy,

- $H T^{\prime}>500 \mathrm{GeV}$,

- $\mathbb{E}_{T}>400 \mathrm{GeV}$.

The resulting cut flow is displayed in table 7. As advertised, the number of leptonic events to survive the cuts is not significantly above the SM background, whereas a significant number of events survive in HH50 and HH50' (cf. figure 3). This set of cuts therefore serves to discriminate between our model and CMSSM-like models.

\subsection{Model discrimination: a simplified model}

The analysis of section 5.2 relies on the presence of intermediate states (in the case of HH50 and HH50', the wino-like $\chi_{1}^{ \pm}$and $\chi_{2}^{0}$ ) whose decay into the LSP produces isolated leptons. In models with non-unified gaugino masses, the LSP could still be bino-like while all remaining charginos and neutralinos are much heavier. Can we still distinguish our 


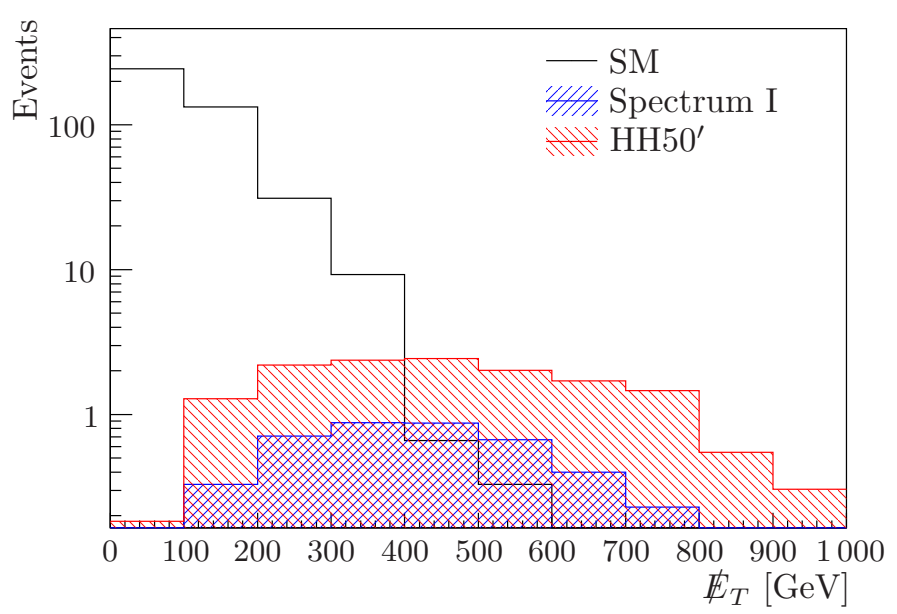

Figure 3. $\mathbb{E}_{T}$ distribution in the semi-leptonic analysis before the final $\mathbb{E}_{T}$ cut. SM events are black, events in Spectrum I are blue and events in HH50' are red.

model from a generic model with a comparably heavy $\tilde{t}_{1}$ and only a light bino LSP below it? It turns out that this is rather more difficult, but still possible.

The simplified model in table 1 has been designed to reproduce the relevant collider signals. We use the production cross section of stop pairs taken from Spectrum I. The only active states are a moderately heavy $\tilde{t}_{1}$ and a light bino-like $\chi_{1}^{0}$. Stops that are produced in pairs will decay as $\tilde{t}_{1} \rightarrow t \chi_{1}^{0}$, with the $t$ further decaying into $b W$. The signature is therefore $b$-jets and missing energy. A similar decay chain is also open in our model (as in the lower branch in figure 2). However, in our model about $50 \%$ of the stops will decay directly into $b$ quarks and missing energy (as in the upper branch). These latter events will produce slightly harder $b$-jets than those involving an intermediate top.

To select the stop pair production channel in our model, we impose a series of simple cuts. At the pre-selection cut level, we select event with at least two and at most four high-energetic jets with $p_{T}$ larger than $100 \mathrm{GeV}$, similar to the all-hadronic analysis:

- $1<N(j)<5$, where $p_{T}(j)>100 \mathrm{GeV}$,

- $\mathbb{E}_{T}>50 \mathrm{GeV}$.

Heavy squarks and gluinos will decay via long decay chains, typically giving rise to a large number of high-energetic jets. Therefore, we select events with exactly two highenergetic jets in order to single out stop pair production. Furthermore, we demand that at least one of these jets is a $b$-jet:

- $N(j)=2$, where $p_{T}(j)>100 \mathrm{GeV}$,

- $N(b$-jets $) \geq 1$.

The invariant mass of the 2-jet system originating in such decays is sensitive to the masses of the parent particles. We select events with relatively small 2-jet transverse mass:

- $m_{j j}^{T} \equiv \sqrt{2 p_{T}\left(j_{1}\right) p_{T}\left(j_{2}\right)\left(1-\cos \Delta \phi\left(j_{1}, j_{2}\right)\right)}<500 \mathrm{GeV}$ 


\begin{tabular}{|c|c|c|c|c|c|c|c|c|c|c|c|}
\hline & \multirow{2}{*}{$\begin{array}{l}\text { before } \\
\text { cuts }\end{array}$} & \multicolumn{2}{|c|}{ pre-cuts } & \multirow[b]{2}{*}{$N(j)$} & \multirow[b]{2}{*}{$b$-tag } & \multirow[b]{2}{*}{$m_{j j}^{T}$} & \multirow[b]{2}{*}{$H T^{\prime}$} & \multirow[b]{2}{*}{$\Delta \phi$} & \multirow[b]{2}{*}{$\mathbb{E}_{T}$} & \multirow[b]{2}{*}{$N(l)$} \\
\hline & & & $N(j)$ & $\mathbb{E}_{T}$ & & & & & & & \\
\hline \multirow{7}{*}{ 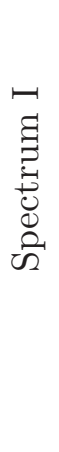 } & $\tilde{q} \tilde{q}^{*}$ & 720 & 569 & 555 & 71 & 12 & 3 & 3 & 2 & 2 & 2 \\
\hline & $\tilde{q} \tilde{q}$ & 7660 & 6416 & 6329 & 978 & 179 & 55 & 53 & 48 & 33 & 24 \\
\hline & $\tilde{t} \tilde{t}^{*}$ & 11220 & 8909 & 8729 & 6093 & 3158 & 1928 & 1378 & 1238 & 637 & 575 \\
\hline & $\tilde{b} \tilde{b}^{*}$ & 1200 & 993 & 983 & 651 & 332 & 152 & 125 & 116 & 72 & 63 \\
\hline & $\tilde{g} \tilde{g}$ & 5800 & 6478 & 4622 & 658 & 348 & 144 & 115 & 104 & 78 & 58 \\
\hline & $\tilde{g} \tilde{q}$ & 16900 & 13425 & 13257 & 1803 & 684 & 243 & 201 & 178 & 121 & 77 \\
\hline & events & & & & & & & & & & 8 \\
\hline \multirow{2}{*}{\multicolumn{2}{|c|}{$\begin{array}{r}\text { simplified } \\
\text { events }\end{array}$}} & 11220 & 8179 & 7986 & 5328 & 2107 & 1339 & 782 & 666 & 316 & 243 \\
\hline & & & & & & & & & & & 2 \\
\hline \multirow{3}{*}{$\sum_{\tilde{S}}$} & $t \bar{t}$ & $1 \times 10^{7}$ & $3 \times 10^{6}$ & $1 \times 10^{6}$ & 739752 & 290416 & 268254 & 34062 & 8669 & 34 & 16 \\
\hline & $t$ & $1.7 \times 10^{6}$ & 160197 & 23773 & 21234 & 6858 & 6330 & 907 & 176 & 6 & 3 \\
\hline & events & & & & & & & & & & 8 \\
\hline
\end{tabular}

Table 8. Cut flow of the analysis in which we examine the possibility to distinguish $\tilde{t}$ decays via bino-like neutralinos from decays via higgsino-like neutralinos at $\sqrt{s}=7 \mathrm{TeV}$. The remaining signal and background events, scaled to an integrated luminosity of $20 \mathrm{fb}^{-1}$, are printed in bold.

In order to suppress the Standard Model background we employ following cuts:

- $H T^{\prime}>400 \mathrm{GeV}$,

- $\Delta \phi\left(\mathbb{E}_{T}, j_{2}\right)>1$,

- $\mathbb{E}_{T}>400 \mathrm{GeV}$,

- $N(l)=0$.

Missing transverse energy in QCD di- and multi-jet events can only appear due to the mismeasurement of one of the jets. We assume that, in events with very large missing transverse energy and exactly two high-energetic jets, the mismeasured jet is the next-toleading one. We therefore expect that no QCD event will survive the cuts on $\Delta \phi\left(\mathbb{E}_{T}, j_{2}\right)$ and $\mathbb{E}_{T}$. The resulting cut flow is displayed in table 8 .

Evidently, these cuts can discriminate between Spectrum I and the simplified model. Of course the latter is not a realistic scenario, and in a fully-fledged model cascade decays of heavier states may also be relevant. However, since the cuts single out the stop pair production channel in our model quite efficiently, it seems reasonable to expect that this remains true for a generic full model which the simplified model is taken to represent here. The cuts are even tight enough to remove almost all of the stop decay events in the simplified model, while leaving a substantial excess above the Standard Model background 
in our model (presumably coming from direct $\tilde{t}_{1} \rightarrow b \chi_{1}^{ \pm}$decays). Note, however, that this analysis will be rather challenging with real data: Only few events survive, and the discrimination is not mainly due to a single cut, but rather to the combined effects of all of them.

\section{Conclusions}

Supersymmetry could still be just around the corner, even though the corner has now moved a bit. Should candidate SUSY signatures be observed within the next year, it will be interesting to see how much can already be found out about the underlying model at the early LHC. It is therefore important to study the collider characteristics of physically well motivated scenarios. This is a complementary approach to just studying simplified models, designed for their simple collider phenomenology, or simple ad-hoc parametrizations such as the CMSSM.

In this paper we have investigated the early-LHC prospects for the MSSM with light higgsinos, as obtained from simple mixed gauge-gravity mediated models which are motivated by a certain class of string compactifications. For a first tentative study of their collider phenomenology, we have analyzed a particular benchmark point in some detail. With early LHC data, evidence for our model could be found in jets plus missing transverse energy searches. Moreover, with dedicated cuts and using also the leptonic search channels, it will be possible to distinguish our model from more commonly studied standard SUSY frameworks, such as the CMSSM or a bino-LSP simplified model.

Our analysis is rather crude compared to what could be done with a full detector simulation and using state-of-the-art multivariate analysis methods. We have also restricted ourselves to a best-case scenario with a favourable choice of parameters. The present paper should therefore be regarded as a first step to the exploration of our class of models. It would be very interesting to refine and extend this study to the full parameter space, as far as it can be explored by early LHC, at least once there are hints for supersymmetry. Furthermore, it should be worthwile to also assess the discovery potential at $14 \mathrm{TeV}$.

\section{Acknowledgments}

The authors thank A. Bharucha, S. Brensing, T. Figy, C. Sander, and P. Schleper for useful discussions.

Open Access. This article is distributed under the terms of the Creative Commons Attribution License which permits any use, distribution and reproduction in any medium, provided the original author(s) and source are credited.

\section{References}

[1] F. Brummer and W. Buchmüller, Light higgsinos as heralds of higher-dimensional unification, JHEP 07 (2011) 010 [arXiv:1105.0802] [INSPIRE]. 
[2] H. Baer, V. Barger and P. Huang, Hidden SUSY at the LHC: the light higgsino-world scenario and the role of a lepton collider, JHEP 11 (2011) 031 [arXiv:1107.5581] [INSPIRE].

[3] S. Bornhauser, M. Drees, S. Grab and J. Kim, Light stop searches at the LHC in events with two b-jets and missing energy, Phys. Rev. D 83 (2011) 035008 [arXiv:1011.5508] [InSPIRE].

[4] M. Papucci, J.T. Ruderman and A. Weiler, Natural SUSY endures, arXiv:1110.6926 [INSPIRE].

[5] X.-J. Bi, Q.-S. Yan and P.-F. Yin, Probing light stop pairs at the LHC, arXiv:1111.2250 [INSPIRE].

[6] N. Desai and B. Mukhopadhyaya, Constraints on supersymmetry with light third family from LHC data, arXiv: 1111.2830 [INSPIRE].

[7] C. Brust, A. Katz, S. Lawrence and R. Sundrum, SUSY, the third generation and the LHC, arXiv:1110.6670 [INSPIRE].

[8] G.L. Kane, Perspectives on supersymmetry, World Scientific, Singapore (1998).

[9] C. Csáki, A. Falkowski, Y. Nomura and T. Volansky, New approach to the $\mu$-B $\mu$ problem of gauge-mediated supersymmetry breaking, Phys. Rev. Lett. 102 (2009) 111801 [arXiv: 0809.4492] [INSPIRE].

[10] A. De Simone, R. Franceschini, G.F. Giudice, D. Pappadopulo and R. Rattazzi, Lopsided gauge mediation, JHEP 05 (2011) 112 [arXiv:1103.6033] [INSPIRE].

[11] Z. Lalak, S. Pokorski and K. Turzynski, Gravity in gauge mediation, JHEP 10 (2008) 016 [arXiv: 0808.0470] [INSPIRE].

[12] B. Allanach, SOFTSUSY: a program for calculating supersymmetric spectra, Comput. Phys. Commun. 143 (2002) 305 [hep-ph/0104145] [InSPIRE].

[13] J. Alwall et al., MadGraph/MadEvent v4: the new web generation, JHEP 09 (2007) 028 [arXiv:0706.2334] [INSPIRE].

[14] T. Sjöstrand, S. Mrenna and P.Z. Skands, PYTHIA 6.4 physics and manual, JHEP 05 (2006) 026 [hep-ph/0603175] [INSPIRE].

[15] J. Pumplin et al., New generation of parton distributions with uncertainties from global QCD analysis, JHEP 07 (2002) 012 [hep-ph/0201195] [INSPIRE].

[16] M. Mühlleitner, SDECAY: a Fortran code for SUSY particle decays in the MSSM, Acta Phys. Polon. B 35 (2004) 2753 [hep-ph/0409200] [INSPIRE].

[17] S. Ovyn, X. Rouby and V. Lemaitre, DELPHES, a framework for fast simulation of a generic collider experiment, arXiv:0903.2225 [INSPIRE].

[18] N. Kidonakis, Top quark pair and single top production at Tevatron and LHC energies, PoS (ICHEP 2010) 059 [arXiv: 1008.2460] [INSPIRE].

[19] J.M. Campbell, R. Ellis and C. Williams, Vector boson pair production at the LHC, JHEP 07 (2011) 018 [arXiv:1105.0020] [INSPIRE].

[20] P. Torrielli and S. Frixione, Matching NLO QCD computations with PYTHIA using MC@NLO, JHEP 04 (2010) 110 [arXiv: 1002.4293] [INSPIRE].

[21] L. Randall and D. Tucker-Smith, Dijet searches for supersymmetry at the LHC, Phys. Rev. Lett. 101 (2008) 221803 [arXiv:0806.1049] [INSPIRE].

[22] CMS collaboration, SUSY searches with dijet events, CMS-PAS-SUS-08-005 (2008).

[23] CMS collaboration, Search strategy for exclusive multi-jet events from supersymmetry at CMS, CMS-PAS-SUS-09-001 (2009). 